Bundesgesundheitsbl 2018 - 61:1363-1364 https://doi.org/10.1007/s00103-018-2830-z Online publiziert: 18. Oktober 2018 (c) Springer-Verlag GmbH Deutschland, ein Teil von Springer Nature 2018

CrossMark
Anne Starker · Anke-Christine Saß

Robert Koch-Institut, Berlin, Deutschland

\title{
Wer raucht denn noch? Sind E-Zigaretten wirklich ungefährlich? Wie funktionieren Tabakerhitzer?
}

Diese und ähnliche Fragen tauchen auf, wenn man sich mit Freunden und Bekannten über das Thema Rauchen unterhält. Neue Entwicklungen beim Tabakkonsum waren der Ausgangspunkt für die Konzeption des nun vorliegenden Schwerpunktheftes. Aus Public-HealthPerspektive bleibt Tabakrauchen allerdings - trotz sinkender Prävalenzen und neuer Produkte - ein bedeutender und vermeidbarer Risikofaktor für zahlreiche chronische Erkrankungen, wie Herz-Kreislauf-Erkrankungen, Atemwegserkrankungen und Krebs. Rauchen zählt in Deutschland weiterhin zu den führenden Ursachen für vorzeitige Sterblichkeit. Das Risiko betrifft aber nicht nur Raucherinnen und Raucher, sondern auch Personen, die Passivrauch ausgesetzt sind - wenn auch in geringerem Ausmaß. Ein Großteil der Raucherinnen und Raucher konsumiert nach wie vor Tabak in Zigarettenform. Konsumtrends beim Rauchen zeigen aber einen Zuwachs bei elektrischen Zigaretten (E-Zigaretten), vor allem bei Jugendlichen und jungen Erwachsenen. Die Tabakindustrie bewirbt zurzeit stark Tabakerhitzer, bei denen anders als bei E-Zigaretten keine nikotinhaltige Flüssigkeit, sondern echter Tabak erhitzt, aber nicht verbrannt wird. Über das Ausmaß der Schädlichkeit von E-Zigaretten und Tabakerhitzern und ihren Einsatz zur Raucherentwöhnung wird in der Fachwelt und Öffentlichkeit kontrovers diskutiert. Und auch wenn zahlreiche tabakpräventive Maßnahmen sowie Nichtraucherschutzgesetze auf Bundes- und Landesebene ergriffen wurden, erscheinen die bislang erfolgten Maßnahmen im internationalen Vergleich noch ausbaufähig.

Vor diesem Hintergrund sind für das vorliegende Schwerpunktheft Themenbereiche ausgewählt worden, die aktuelle Trends beim Tabakkonsum und die Entwicklungen bei der Tabakprävention beschreiben. Darüber hinaus werden wissenschaftliche Erkenntnisse zu den kontrovers diskutierten Produkten E-Zigarette und Tabakerhitzer zusammengeführt und erörtert. Das Heft gibt damit einen aktuellen Überblick über die verschiedenen Aspekte des Themas Rauchen in Deutschland.

Im ersten Themenbereich werden Trends beim Tabakkonsum beschrieben. Dazu wird zunächst ein Überblick über die zeitlichen Trends beim Rauchen Erwachsener in Deutschland von 1990 bis 2015 gegeben (Zeiher et al.). Der Fokus liegt hierbei auf den Ergebnissen des Gesundheitsmonitorings am Robert Koch-Institut. Im zweiten Beitrag wird die Veränderung des Rauchverhaltens bei Jugendlichen und jungen Erwachsenen beschrieben. Orth und Merkel gehen darin der Frage nach, wie sich die Konsumprävalenzen von Tabakzigaretten, Wasserpfeifen, E-Zigaretten und E-Shishas entwickelt haben. Hierzu werden die Ergebnisse von Repräsentativerhebungen der Bundezentrale für gesundheitliche Aufklärung (BZgA) zum Substanzkonsum herangezogen. Im Beitrag von Kuntz et al. wird die zeitliche Entwicklung der berufsgruppenspezi- fischen Rauchquoten aufgezeigt - ein wichtiger Aspekt bei der Beschreibung sozialer Unterschiede im Rauchverhalten. Dazu werden die letzten fünf Wellen des Mikrozensus mit Informationen zum Rauchverhalten herangezogen. Im letzten Beitrag des ersten Themenbereiches geht es um Trends bei Krebserkrankungen infolge des Rauchens. Wienecke und Kraywinkel untersuchen vor dem Hintergrund der Veränderungen beim Rauchverhalten die Entwicklung tabakassoziierter Krebserkrankungen in Deutschland in den letzten 20 Jahren. Dazu nutzen sie Daten der epidemiologischen Krebsregister in Deutschland, die im Zentrum für Krebsregisterdaten (ZfKD) im Robert Koch-Institut zusammengeführt und auf Bundesebene ausgewertet werden.

Der zweite Themenbereich des Schwerpunktheftes widmet sich speziell $E-Z i$ garetten und Tabakerhitzern. Im ersten Beitrag wird anhand der Daten der Deutschen Befragung zum Rauchverhalten (DEBRA-Studie) die Nutzung von E-Zigaretten und Tabakerhitzern in der Bevölkerung vorgestellt. Außerdem werden Gründe für die Nutzung von E-Zigaretten und die subjektive Risikoeinschätzung von Tabakerhitzern dargestellt (Kotz und Kastaun). Die Einschätzung der Gesundheitsgefahren von E-Zigaretten in verschiedenen Bevölkerungsgruppen ist Gegenstand des zweiten Beitrags (Atzendorf et al.). Anhand der Daten des aktuellen Epidemiologischen Suchtsurveys 2015 wird in diesem Beitrag außerdem untersucht, ob die 
Einschätzung der Gesundheitsgefahren einen Einfluss auf die Nutzung von E-Zigaretten als Mittel zur Tabakentwöhnung hat. Der dritte Beitrag widmet sich Tabakerhitzern. Die Produktklasse wird vorgestellt und auf Grundlage einer Studie des Bundesinstituts für Risikobewertung (BfR) wird ein Vergleich der Emissionen mit denen konventioneller Zigaretten vorgenommen (Pieper et al.).

Der abschließende Themenbereich befasst sich mit der Tabakprävention. Dazu werden im ersten Beitrag die verschiedenen Maßnahmen zur Tabakkontrolle vorgestellt, die seit 2002 in Deutschland durchgesetzt wurden. Anschließend wird die deutsche Tabakpräventionspolitik im internationalen Vergleich eingeordnet (Schaller und Mons). Im nächsten Beitrag werden die deutschlandweiten Maßnahmen der Bundeszentrale für gesundheitliche Aufklärung (BZgA) zur Tabakprävention vorgestellt, die im Rahmen der „rauchfrei“-Kampagne adressatenspezifisch für Jugendliche von 12 bis 17 Jahren und für Erwachsene angeboten werden (Goecke und Duhme). Zwei erfolgreiche Praxisbeispiele der Tabakprävention bilden den Abschluss dieses Themenbereichs: das Projekt „Be Smart - Don't Start“, ein seit 1997 existierender Wettbewerb für rauchfreie Schulklassen, der vom Institut für Therapie- und Gesundheitsforschung Nord koordiniert wird (Isensee und Hanewinkel), und das Netzwerk „Aufklärung gegen Tabak“, in dem Medizinstudierende ehrenamtlich Schülerinnen und Schüler über das Rauchen aufklären. Das Netzwerk qualifiziert auch angehende Ärztinnen und Ärzte, damit sie ihre Patientinnen und Patienten bei einem Rauchausstieg fundiert beraten und begleiten können (Brinker et al.).

In der im Jahr 2010 erschienenen Ausgabe des Bundesgesundheitsblattes zum Thema „Tabakprävention“ resümierten die Herausgeberinnen Ulrike Maschewsky-Schneider und Elisabeth Pott: „Trotz aller Fortschritte zeigt sich deutlich, dass Deutschland in der Tabakprävention gemessen am Anteil Raucher und Raucherinnen in der Bevölkerung - noch lange nicht am Ziel angekommen ist.“ Diese Aussage gilt auch heute noch. Wir hoffen, dass Sie durch das neue
Schwerpunktheft gut über aktuelle Entwicklungen informiert und vielleicht dazu angeregt werden, sich für die Erreichung dieses Ziels (weiterhin) zu engagieren.

Ihre

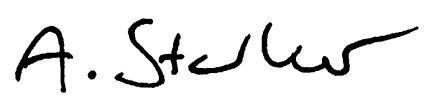

Anne Starker

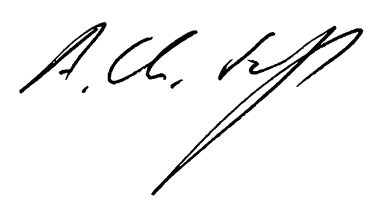

Anke-Christine Saß

\section{Korrespondenzadresse}

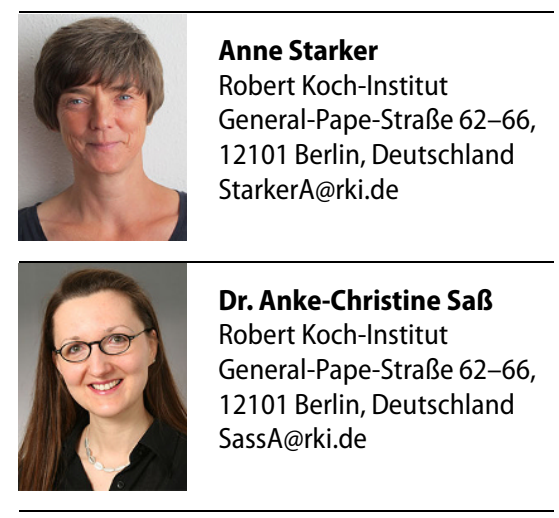

Interessenkonflikt. A. Starker und A.-C. Saß geben an, dass kein Interessenkonflikt besteht. 